\title{
THE ANNEALING EFFECT ON STRUCTURAL AND OPTICAL PROPERTIES OF ZnO THIN FILMS PRODUCED BY RF SPUTTERING
}

\begin{abstract}
A. G. Rolo, J. Ayres de Campos, T. Viseu, T. de Lacerda-Arôso and M.F. Cerqueira
Departamento de Física, Universidade do Minho, Campus de Gualtar 4710-057 Braga, Portugal

In this work, a study of the structure and optical properties of undoped $\mathrm{ZnO}$ thin films produced by r.f. magnetron sputtering technique as a function of the growth parameters is reported. Modification under annealing conditions is also analysed. Raman spectroscopy, $\mathrm{X}$-ray photoelectron spectroscopy, X-ray diffraction and optical transmittance have been used. From the position of the (002) X-ray diffraction peak and the $E_{2}$ (high) mode detected in Raman spectra, the residual stress both in the as-grown and in the annealed samples has been estimated.
\end{abstract}

\section{Keywords: ZnO; Thin films; X-ray; Raman; Stress}

\section{Introduction}

Zinc oxide $(\mathrm{ZnO})$ is a II-VI wide direct-gap semiconductor with a band gap of $3.37 \mathrm{eV}$ at room temperature which makes it interesting for optoelectronic applications in the near ultraviolet (UV) region. Mostly in bulk, $\mathrm{ZnO}$ has been intensively studied for the past fifty years. A renewed interest in $\mathrm{ZnO}$ derived materials arouse in the mid nineties of the last century, due to its potential technological applications. Since then, $\mathrm{ZnO}$ thin films, quantum wells, nano-rods and quantum dots have been produced and studied hopping to apply them in optoelectronics, electronic devices as alternative to ITO and spintronics. In this study, experimental results of crystalline structure, stress, refractive index and chemical composition of $\mathrm{ZnO}$ thin films before and after annealing treatment are discussed.

\section{Experimental}

$\mathrm{ZnO}$ thin films have been grown on glass substrates within a mixture of $\mathrm{O}_{2}$ and Ar gases at a constant working pressure of $0.7 \mathrm{~Pa}$ and a $50^{\circ} \mathrm{C}$ constant substrate temperature, in an 
Alcatel SCM 650 sputtering system. The target consisted of a hyper-pure (99.99\%) metal zinc wafer spaced $60 \mathrm{~mm}$ away from the substrates.

Films thickness and optical parameters have been determined by optical transmittance in near ultra-violet - visible -near infra-red range. The micro-structure of the films has been analysed by X-ray diffraction and Raman scattering. Optical transmittance spectra have been measured in a Shimadzu UV 3101 PC spectrometer. X-ray experiments (BraggBretano geometry) have been performed in a Philips PW 1710 spectrometer using $\mathrm{CuK}_{\alpha}$ radiation. Micro-Raman spectra have been measured on a Jobin-Yvon T64000 spectrometer equipped with a cooled CCD detector, using the $514.5 \mathrm{~nm}$ excitation line of an $\mathrm{Ar}^{+}$laser in the back scattering geometry.

Growth conditions, thickness, refractive index, and structural parameters obtained by Xray are shown in table I.

\section{Results and discussion}

All films are highly transparent in the visible and NIR range, as illustrated by the example in figure 1. Thickness and optical parameters of the films have been determined by fitting the spectra using the Minkov ${ }^{[1]}$ method and adopting the unified treatment of Forouhi and Bloomer ${ }^{[2]}$ for the dispersion behaviour of the dielectric constant. A fit to the experimental spectrum is also shown in figure 1. The thickness of the films ranges from 230 to $830 \mathrm{~nm}$ which can be related to deposition conditions. Unexpectedly, the calculated refractive index of the as-grown films is slightly higher than the bulk value. Assuming as Fang et al ${ }^{[3]}$, that refractive index and packing density are correlated, it may be inferred that the high material density is to be due to the film stress. In order to better understand this behaviour, films have undergone thermal treatment at $500^{\circ} \mathrm{C}$ in air, for three hours. The refractive index dispersion of $\mathrm{ZnO}$ monocrystal ${ }^{[4]}$ and of a typical film, before and after annealing, are depicted in the inset of figure 1. On all annealed samples the refractive index is lower than the bulk value. 
Table 1

Growth conditions, thickness, refractive index (n), and structural parameters obtained by XRD of as-grown (S\#) and annealed (S\#-A) $\mathrm{ZnO}$ thin films

\begin{tabular}{lllllrr}
\hline Sample & $F\left(\mathrm{O}_{2}\right)$ & RF power $(\mathrm{W})$ & Thickness $(\mathrm{nm})$ & $n(633 \mathrm{~nm})$ & $D(\AA)$ & $\sigma(\mathrm{GPa})$ \\
\hline S1 & 0.28 & 50 & 289.2 & 2.03 & 146.6 & -7.9 \\
S2 & 0.13 & 50 & 561.0 & 2.03 & 197.0 & -6.1 \\
S3 & 0.28 & 50 & 342.3 & 2.08 & 178.2 & -6.9 \\
S4 & 0.50 & 50 & 262.4 & 2.04 & 63.0 & -10.9 \\
S5 & 0.62 & 50 & 228.3 & 1.99 & 62.1 & -8.5 \\
S6 & 0.13 & 80 & 826.5 & 2.03 & 108.1 & -7.5 \\
S7 & 0.50 & 80 & 418.1 & 2.08 & 32.2 & -10.2 \\
S8 & 0.23 & 50 & & 2.04 & 69.1 & -11.1 \\
S2-A & & & & 1.95 & 271.2 & -1.0 \\
S4-A & & & & 1.94 & 257.3 & -1.0 \\
S8-A & & & & 2.00 & 246.4 & -1.5 \\
\hline
\end{tabular}

$F\left(\mathrm{O}_{2}\right)=p \mathrm{O}_{2} /\left(p \mathrm{Ar}+p \mathrm{O}_{2}\right)$ means the relative oxygen fraction.

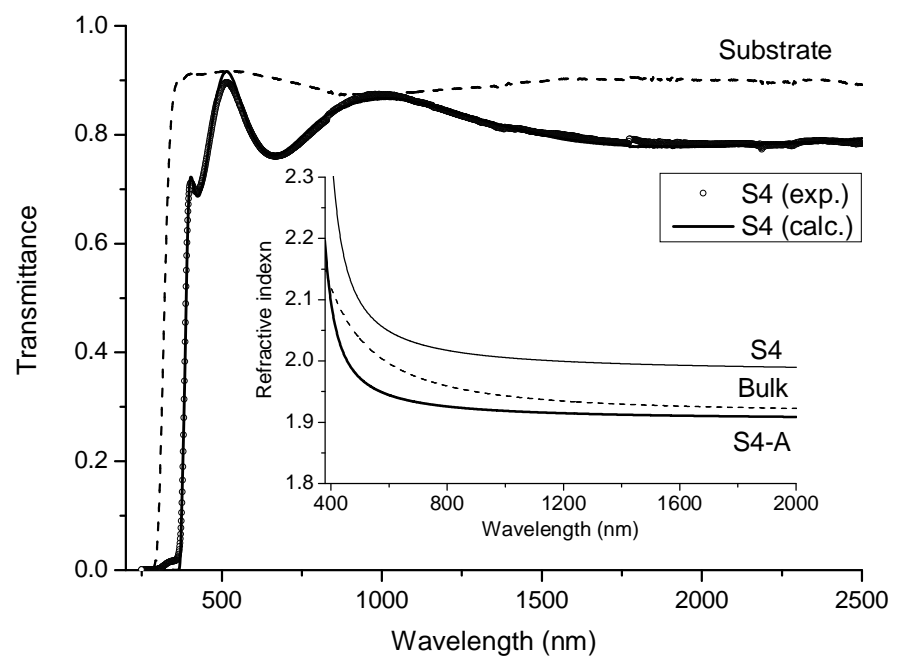

Figure 1 - Transmission spectra (experimental data and fit) of a typical $\mathrm{ZnO}$ sample. Inset shows the refractive index of the $\mathrm{ZnO}$ monocrystal and of the film, before and after annealing.

The X-ray diffraction spectra of $\mathrm{ZnO}$ thin films reveal the existence of a single phase with hexagonal wurtzite structure as in bulk $\mathrm{ZnO}$. Only (002) peak or (002) and (004) 
peaks are observed evincing a highly preferential orientation along the c-axis, perpendicular to the substrate surface, in consonance with literature ${ }^{[5-7]}$.

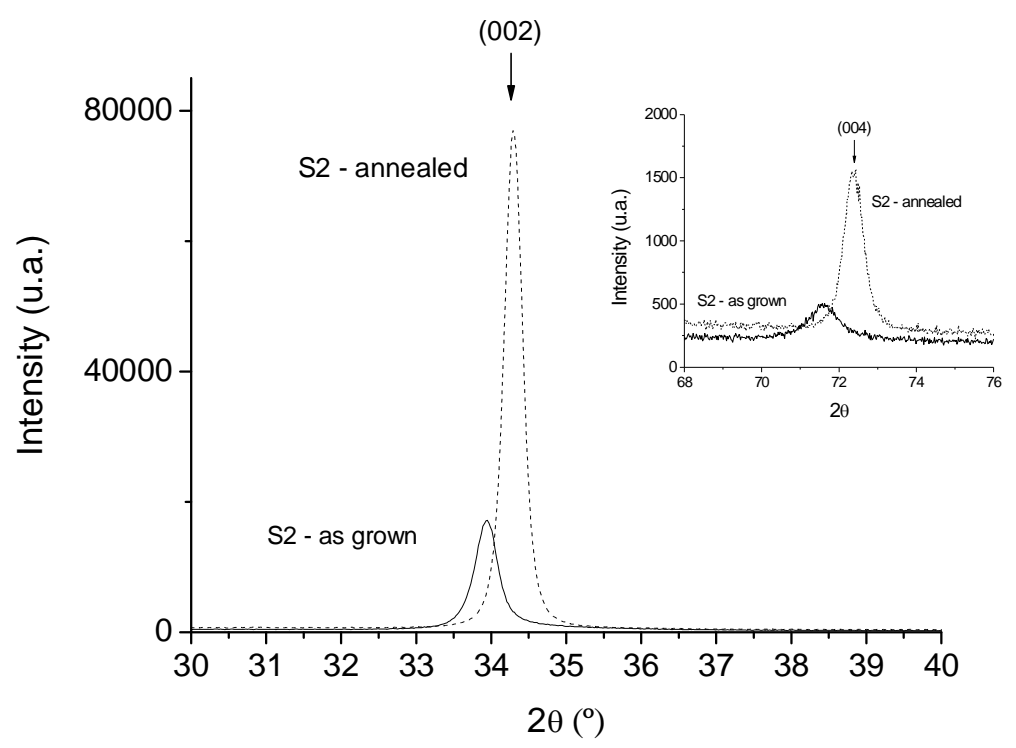

Figure 2 - XRD spectra of a typical ZnO thin film exhibiting (002) and (004) peaks, before and after annealing.

For all as-grown films the (002) peak is asymmetric and shifted from the bulk peak position towards smaller angles, revealing that the structure is stressed. Figure 2 shows the (002) and (004) peaks from the above sample, before and after annealing. With annealing peaks intensity increases and they shift towards the bulk position, demonstrating some improvement in the crystallinity of the film. The (002) peak position as a function of the asymmetry (measured as the ratio between the calculated symmetric curve and the experimental curve) for the as-grown and annealed films is plotted in figure 3. As shown, the asymmetry decreases as the peak position approaches that of the bulk, supporting that the stress has been relief during the thermal treatment. From the XRD data lattice parameter $(c)$, domain size $(D)$, as well as the strain along c-axis $\left(e_{z z}\right)$, and the corresponding stress $(\sigma)$ have been calculated according to the procedure described in literature $^{[5,7-11]}$ (table I). After annealing, all the samples exhibit the same average domain size (around $258 \AA$ ), independently of the as-grown microstructure (table I and figure3). 


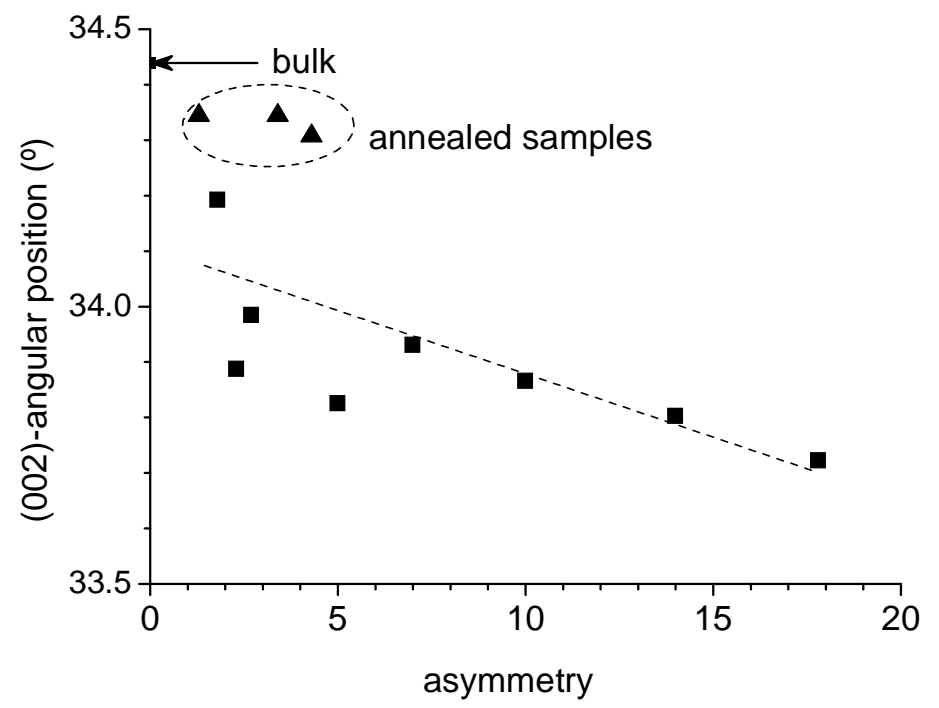

Figure 3 - The (002)-peak position as a function of its asymmetry (see text) for both asgrown and annealed samples.

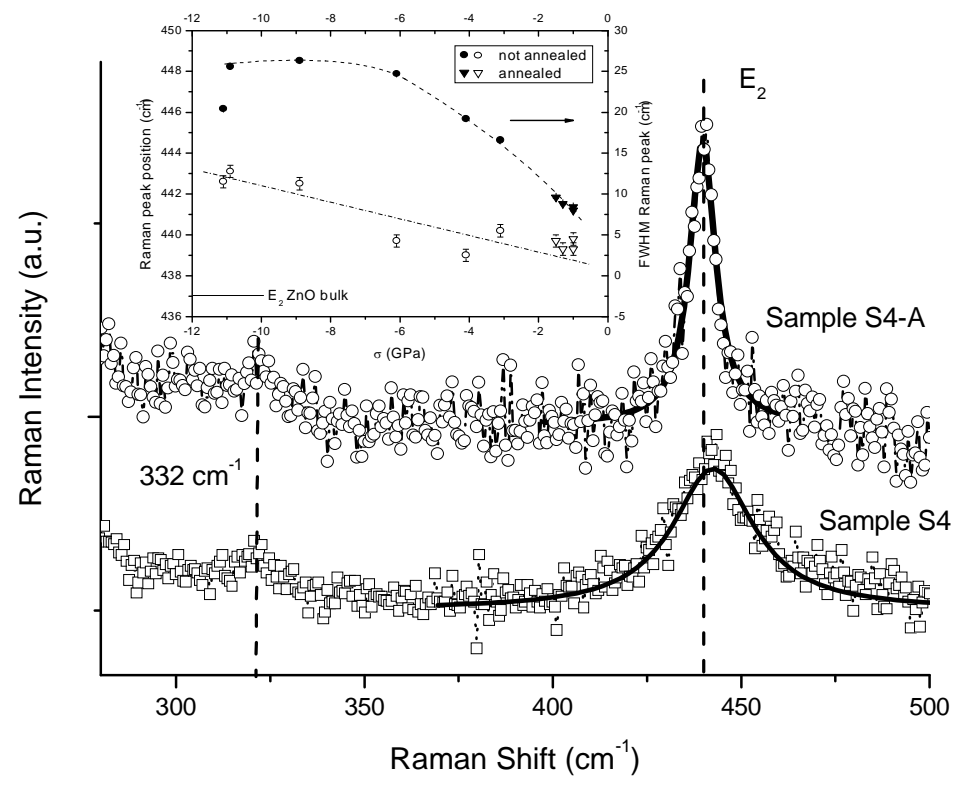

Figure 4 - Typical Raman spectra measured on as-grown and annealed $\mathrm{ZnO}$ films. Full lines represent Lorentzian fits. Inset: $\mathrm{E}_{2}$-peak position and its half-height width 
as a function of stress as assessed by $\mathrm{X}$-ray. Dashed lines are guides for the eyes.

The improvement of the $\mathrm{ZnO}$ crystallinity due to the annealing treatment has also been confirmed by Raman scattering. Figure 4 depicts a typical Raman spectra measured on both as-grown and annealed $\mathrm{ZnO}$ films. The experimental peak at about $435 \mathrm{~cm}^{-1}$ has been assigned to the non-polar optical phonons $\left(\mathrm{E}_{2}\right.$-high) mode ${ }^{[12]}$. After annealing, the intensity of this mode increases, the peak position shifts $\left(2.0-5.5 \mathrm{~cm}^{-1}\right)$ towards the bulk position and the full width at half maximum (FWHM) decreases $\left(26.6\right.$ to $8.4 \mathrm{~cm}^{-1}$ ), as it can be seen in the inset of Figure 4. This behaviour suggests a compressive stress in the non-annealed samples. The peak at $332 \mathrm{~cm}^{-1}$, which can be seen in the Raman spectra both on the as-grown and on the annealed samples, has been assigned to multiple phonon scattering processes ${ }^{[13]}$. The Raman measurements have thus proven to be a sensitive probe to stress relaxation and stoichiometry improvement in the film.

\section{Final remarks}

Undoped $\mathrm{ZnO}$ thin films have been grown by reactive magnetron r.f. sputtering under different $\mathrm{Ar} / \mathrm{O}_{2}$ atmospheres. Films with different crystal sizes, ranging from $3.2 \mathrm{~nm}$ to $19.7 \mathrm{~nm}$ in the as-grown form, have been obtained by changing the deposition parameters. The reported results have shown that the thermal treatment has conduced to a decrease of the refractive index from above to below the bulk value and to the improvement of film crystallinity. Since the refractive index and packing density are correlated, we have assumed that the high material density in the as-grown films is to be due to the film stress. This compressive stress has been relieved by annealing as it has been confirmed by our X-ray and Raman data.

\section{References}

[1] - D. A. Minkov, J. Phys D: Appl. Phys. 22 (1989) 199

[2] - A. R. Forouhi, I. Bloomer, Phys. Rev B 34 (1986) 7018

[3] - Z.B. Fang, Z.J. Yan, Y.S. Tan, X.Q. Liu, Y.Y. Wang, Appl. Surface Science, 241 (2005) 303

[4] - S. H. Wemple, M DiDomenico, J. Phys Rev B 3 (1971) 1338 
[5] - S. Maniv, W. D. Westwood, E. Coloboni, J. Vac. Sci. Techonol. 20 (1982) 162

[6] - American Standard for Testing of Materials (ASTM), vol.36 p.145.

[7] - Th. H. de Keijser, J. I. Langford, E. F. Mittemeyer and A. B. P. Rogels, J. Appl. Crystallogr. 11 (1978) 10

[8] - H. P. Klug and L. E. Alexander, "X-ray Diffraction Procedures for Polycrystalline and Amorphous Materials", J. Willey and sons, New York, $2^{\text {nd }}$ ed. (1974) cap.9

[9] - O. Zhenxing, Z. Xiaozhong, Z. Mingzhou, W. Xizhong, L. Yujin, IEEE Trans. Sonics Ultrason. SU-32 (1985) 630

[10] - R.R. Reeber, J. Appl. Phys. 41 (1970) 5063

[11] - T. B. Bateman, J. Appl. Phys., 35 (1962) 3309

[12] - B. H. Bairamov, A. Heinrich, G. Irmer, V. V. Toporov, and E. Ziegler, Phys. Status Solidi B 119 (1983) 227

[13] - T. C. Damen, S. P. S. Porto, and B. Tell, Phys. Rev. 142 (1966) 570 AND J. M. Calleja and M. Cardona, Phys. Rev. B 16 (1977) 3753 